ERRAT UM

R. Yaka $\cdot$ U. Yinon $\cdot$ M. Rosner $\cdot$ Z. Wollberg

\title{
Pathological and experimentally induced blindness induces auditory activity in the cat primary visual cortex
}

Published online: 13 April 2000

(C) Springer-Verlag 2000

\section{Exp Brain Res (2000) 131:144-148}

On page 145, at the end of the Materials and methods section, an incorrect statement was printed to the effect that all procedures were performed in accordance with the guidelines issued by the "Tel Aviv University Animal Care and Use Committee." Although all procedures were appropriate, this commitee had not yet been established when the above study was carried out. The statement should therefore be deleted.

The online version of the original article can be found at http://dx.doi.org/10.1007/s002219900295

R. Yaka $\cdot$ Z. Wollberg (

Department of Zoology,

The George. S. Wise Faculty of Life Sciences,

Tel-Aviv University, Tel-Aviv 69978, Israel

e-mail: wollberg@ccsg.tau.ac.il

Tel.: +972-3-6409124, Fax: +972-3-6409403

R. Yaka $\cdot$ U. Yinon $\cdot$ M. Rosner

Goldschleger Eye Research Institute, Sheba Medical Center,

Tel-Hashomer, The Sackler Faculty of Medicine,

Tel-Aviv University, Tel-Aviv 69978, Israel 